\title{
Efecto de reciprocidad y retroalimentación audiovisual en una tendencia cooperativa o egoísta
}

Effect of reciprocity and audiovisual feedback on a cooperative or selfish tendency

Efeito da reciprocidade e do feedback audiovisual numa tendência cooperativa ou egoísta

Juan Sánchez*, \& Diego Mauricio Otálora Morales

Universidad del Bosque

Citar este artículo así:

Sánchez J., \& Otálora, D. M. (2020). Efecto de reciprocidad y retroalimentación audiovisual en una tendencia cooperativa o egoísta. Revista Enfoques, 3(1), 45-6o. http://dx.doi.org/

Derechos de autor: Licencia Creative Commons AtribuciónNoComercial-SinDerivadas 4.0 internacional y 2.5 Colombia (CC BYNC-ND 2.5 (O)

Recibido: febrero 16 de 2018

Revisado: mayo 03 de 2018 Aceptado: noviembre 21 de 2018

* Contacto: Juan Sánchez, email: jmsanchezd95@gmail.com ORCID: 0000-0001-9598-2657 
Resumen | El objetivo del presente estudio es identificar el efecto de la presentación de vídeos que promueven conducta prosocial o egoísta, luego de haber desarrollado una tendencia a comportarse de forma altruista o egoísta en la tarea experimental del dilema del prisionero. 70 estudiantes universitarios fueron expuestos a dos modalidades del dilema del prisionero de 50 ensayos, con el fin de inducir dos tendencias, una prosocial y otra egoísta. Posterior a los 50 ensayos, se presentó un vídeo que promovía la conducta opuesta a la tendencia previamente desarrollada y finalmente se realizó un último ensayo del dilema del prisionero. Los resultados indican que existe un efecto estadísticamente significativo de reciprocidad en la tarea del dilema del prisionero. No se observó una relación significativa entre la presentación del vídeo y el cambio en la tendencia previamente desarrollada.

Palabras claves / Juego del dilema del prisionero; Conducta Prosocial; Reciprocidad.

Abstract | The objective of this study is to identify the effect of the presentation of videos that promote prosocial or selfish behavior after having developed a tendency to behave in an altruistic or selfish way in the experimental task of prisoners' dilemma. 70 university students were exposed to two modalities of the prisoner's dilemma of 50 trials, to induce two tendencies, a prosocial and a selfish one. After the 50 trials, a video that promoted the behavior opposite the previously developed trend was presented, and finally a prisoners' dilemma one-shot was made. The results indicate that there is a statistically significant effect of reciprocity on the task of the prisoner's dilemma. No significant relationship between video presentation and change in the previously developed trend was observed.

Keywords / Prisoners Dilemma Game; Prosocial Behavior; Reciprocity.

Resumo / O objetivo deste estudo é identificar o efeito da apresentação de vídeos que promovam o comportamento prosocial ou egoísta depois de ter desenvolvido uma tendência a se comportar de forma altruísta ou egoísta na tarefa experimental do dilema do prisioneiro. 70 estudantes universitários foram expostos a duas modalidades do dilema do prisioneiro de 50 julgamentos, a fim de induzir duas tendências, uma prosocial e uma egoísta. Após o 50 trials, um vídeo foi apresentado que promoveu o comportamento oposto à tendência previamente desenvolvida e, finalmente, um julgamento final do dilema do prisioneiro foi feito. Os resultados indicam que há um efeito estatisticamente significativo da reciprocidade na tarefa do dilema do prisioneiro. Nenhuma relação significativa foi observada entre a apresentação do vídeo e a mudança na tendência previamente desenvolvida.

Palavras-chave / jogo do dilema do prisioneiro; conduta pró social; reciprocidade.

$€ \| 46$ 


\section{Introducción}

El ser humano es un ser social. Todo evento humano tiene por necesidad un matiz social indisoluble que le imprime siempre un tono relativo a la responsabilidad, por lo que todas sus acciones deben (o al menos deberían) encaminarse hacia la convivencia (Corral, 2012). El ser humano ha creado y se ha creado dentro de cultura, instituciones, leyes y otros elementos, cuyo objetivo principal es asegurar una vida en comunidad (Ribes-Iñesta, 2010).

Es necesario resaltar que, ante tal situación, surge el problema que en diferentes circunstancias el bien común puede estar en conflicto con el bien individual; en esos casos se hace difícil contribuir a los dos ámbitos simultáneamente (Attari, Krantz \& Weber, 2014; Baum, Paciotti, Richnerson, Lubell \& McElreath, 2012; O’brien, 2012; Redondo, Rueda \& Amado, 2013; Ribes-Iñesta, et al., 2006; Ribes-Iñesta, et al., 2008; Thøgersen, 2008; Van Miltenburg, Buskens, Barrera \& Raub, 2014). Este problema envuelve una característica particular: el bien común tiende a tener efectos que acaecen sólo a largo plazo, lo que se contrasta con los efectos inmediatos del bien individual.

Un factor que afecta la probabilidad de preferir actuar por el beneficio colectivo o por el beneficio individual, es el comportamiento que otros individuos tienen hacia la persona. RibesIñesta (2010), sostiene que en momentos donde existen contingencias compartidas, es decir, situaciones en las cuales lo que a un individuo le ocurra depende tanto del comportamiento propio como de lo que hagan otras personas, se tiende a desarrollar una relación de reciprocidad que puede ser analizada dentro de un continuo que va desde comportamiento egoísta hasta comportamiento altruista. De acuerdo con esto, el individuo se comportará de forma egoísta si los otros se comportan de forma egoísta con él (reciprocidad negativa); en cambio, si está expuesto a una persona que se comporta de forma prosocial se tenderá a comportar de forma prosocial (reciprocidad positiva), también denominada relación recíproca cooperativa (Redondo, Rueda \& Amado, 2013; Silva, 2014).

En diferentes estudios se ha evidenciado esta relación de reciprocidad. En tareas en las que los sujetos se exponen a contingencias compartidas, se ha encontrado que participantes al ser informados que algunos de sus compañeros en dilemas sociales actuarían de forma egoísta, disminuyeron significativamente la conducta prosocial (Kerr, et al., 2009). El primer 
objetivo del presente estudio consiste en identificar, mediante el dilema del prisionero, el desarrollo o no de comportamiento recíproco con relación al comportamiento del compañero.

Los resultados de los estudios mencionados han mostrado que la reciprocidad se tiende a observar en ensayos iterados en diferentes dilemas sociales. Cabe la pregunta de qué factores podrían afectar una tendencia de comportamiento desarrollada en este tipo de situaciones. En años recientes ha existido un creciente interés en cuanto a la presentación de videos con contenido de tipo violento o cooperativo y sus efectos sobre el comportamiento egoísta y prosocial; el interés se ha suscitado debido al contacto constante del ser humano en su vida cotidiana con estos, en especial en niños y adolescentes (Bandura, Ross \& Ross, 1963; Hogan, 2012).

En diversos estudios con vídeos de tipo prosocial se ha encontrado que la presentación de medios audiovisuales puede tener efectos en el comportamiento prosocial. Prot, et al. (2013) estudiaron la relación entre ver videos con contenido prosocial y violento. Los resultados indicaron que altos niveles de conductas prosociales están relacionados con ver videos de contenido prosocial. Se han encontrado resultados similares en un estudio longitudinal, en donde se buscaba estudiar la relación entre ver programas televisivos con contenido prosocial y el comportamiento prosocial y agresivo, se evidenció una asociación negativa entre programas televisivos con contenido prosocial y el comportamiento agresivo y una asociación positiva entre videos con contenido prosocial y la presentación de esta clase de comportamiento (Padilla-Walker, Coyne, Collier \& Nielson, 2015). Estos resultados sugieren que la presentación de videos que promuevan comportamiento prosocial afecta positivamente la presentación de este comportamiento. En diferentes entornos de distintas ciudades en el mundo se presentan videos que promueven el comportamiento prosocial. Sin embargo, la revisión teórica no permite identificar estudios de corte experimental sobre el efecto de la presentación de estos videos sobre el cambio o no de una tendencia a comportarse de forma egoísta, por tal razón, en la presente investigación se pretende promover a través del efecto de reciprocidad una tendencia a comportarse de forma egoísta en el dilema del prisionero y, posteriormente, observar el efecto de la introducción de un video de personas comportándose prosocialmente sobre el cambio o no, de la mencionada tendencia. El segundo objetivo del presente estudio consiste en identificar dicho efecto. 
Por otra parte, diferentes medios audiovisuales, implícita o explícitamente, promueven comportamiento violento. Se han hecho estudios indagando sobre el papel de videos que promueven este comportamiento. Los estudios realizados, con respecto a vídeos violentos, han demostrado que dichos videos están relacionados positivamente con el comportamiento agresivo, por ejemplo, Quiros \& Geovanna (2016) afirmaron que la televisión influye y estimula la conducta agresiva en los niños, dando como resultado la adopción de estas conductas adversas. Otro ejemplo son los cambios que ocurren a corto plazo en el comportamiento agresivo después de ver películas violentas: se ha evidenciado que personas expuestas a una película violenta presentan mayor comportamiento agresivo en comparación con quienes no son expuestos a una película no violenta (Cruz, 2014; Hoffman, 2014; Qian \& Da-Jun, 2014).

Así como existen estudios que han mostrado correlaciones entre la exposición de videos violentos y comportamientos agresivos, también se han reportado estudios que indican que las presentaciones de videos con contenido violento no promueven dicha clase de comportamiento. Brummert \& Warburton (2011) buscaron comparar el efecto de la exposición a media auditiva y a media visual, de tipo violento, en el comportamiento de agresión. Las personas fueron expuestas a letras de canciones, videos musicales y tonos musicales, violentos y no violentos. No se encontraron diferencias significativas entre los participantes que vieron videos musicales violentos y los que no; sin embargo, la condición en la que se evidenció mayores niveles de comportamiento agresivo fue en la que se presentó un video violento junto con las letras de la canción. Además, los participantes que no fueron expuestos a ningún tipo de media violenta obtuvieron los menores niveles de comportamiento agresivo.

El comportamiento agresivo puede ser visto como comportamiento egoísta, en la medida en que la persona busca el propio bienestar a costa del bienestar de otros (egoísmo extremo) (Chen, Wu, Chen, Wang \& Cen, 2001; Hoyos, 2013). Es plausible suponer que los resultados que se han encontrado sobre el efecto de videos violentos sean extrapolables a los efectos de videos que promueven comportamiento egoísta. Sin embargo, Ramos, Ferguson y Frailing (2014) realizaron un estudio cuyo objetivo era establecer los efectos de videos con contenido violento en la conducta prosocial por medio de una tarea del dilema del prisionero. Los resultados indicaron que no hubo un efecto significativo de los videos violentos en la conducta prosocial. Los autores sostienen como una limitación de su estudio la posibilidad de que el video que utilizaron no representara la variable violencia y el dilema del prisionero 
considera el acto de traicionar como una conducta egoísta, no necesariamente violenta. La revisión teórica no permite identificar estudios de corte experimental sobre la presentación de videos que promuevan comportarse de forma egoísta en el cambio o no, en una tendencia a comportarse de forma prosocial. En la presente investigación se pretende promover a través del efecto de reciprocidad una tendencia a cooperar en el dilema del prisionero y luego observar el efecto de la introducción de un video que promueve comportarse de forma egoísta sobre el cambio o no, de la mencionada tendencia. El tercer objetivo del estudio se orienta a identificar tal efecto.

Teniendo en cuenta que en la introducción se explica cómo los estudios se enfocan únicamente en identificar el efecto que tienen los diferentes contenidos audiovisuales o, por otro lado, identificar el efecto de reciprocidad en diversas tareas de dilemas sociales, cabe resaltar que la diferencia del presente estudio recae en la implementación de ambas variables en un solo diseño. Tanto el dilema del prisionero, para identificar el efecto de reciprocidad, como los vídeos para identificar su efecto sobre una tendencia de comportamiento.

A manera de síntesis el presente estudio tiene tres objetivos, a saber: (a) identificar el efecto de reciprocidad a partir del comportamiento egoísta/prosocial de un confederado sobre el comportamiento individual en ensayos reiterados del dilema del prisionero; (b) identificar el efecto de la presentación de un video con contenido prosocial sobre el cambio o no de una tendencia a comportarse de forma egoísta; y (c) identificar el efecto de la presentación de un video con contenido egoísta sobre el cambio o no de una tendencia a comportarse de forma prosocial.

\section{Método}

\section{Participantes}

Participaron en el estudio de manera voluntaria y anónima, dando de forma escrita su consentimiento, 69 estudiantes de sexo masculino $(n=12)$ y femenino $(n=57)$, con edades comprendidas entre los 18 y los 41 años $(M=22 ; D T=5,1)$, estudiantes universitarios pertenecientes a la Facultad de Psicología de una universidad privada de la ciudad de Bogotá D.C. La muestra total inicial estuvo conformada por 71 participantes, 2 fueron excluidos por presentar problemas con el software durante la toma de datos. 


\section{Instrumentos}

Se diseñó un software basado en el dilema del prisionero a partir de la herramienta Unity. Este dilema consiste en una situación en la cual los participantes tienen la opción de cooperar o traicionar a un compañero real o confederado, los participantes obtienen más o menos puntos en función del comportamiento propio y del comportamiento de un compañero (Devilly, Brown, Pickert \& O’Donohue, 2015; Kanazawa \& Fontaine, 2013; Parra-García, Nieto \& Sánchez-Carrasco, 2014).

Inicialmente el software le presenta al participante una serie de instrucciones, por medio de las cuales se le explica que tendrá que optar por cooperar o traicionar a un compañero aleatorio (pero en realidad el compañero es un confederado) y se le da a conocer los puntos que obtendrá con base en su comportamiento y el de su compañero. Si un compañero traiciona y el otro coopera (traición exitosa), el que traicionó obtiene la mayor cantidad de puntos posibles (5 puntos) y el otro obtiene la menor cantidad de puntos (0 puntos). En el caso de que ambos tomen la misma decisión sea cooperar o traicionar, ambos obtendrán la misma cantidad de puntos. Si ambos cooperan, obtendrán mayor número de puntos (3 puntos cada uno) que si ambos traicionan (1 punto para cada uno) (Ver figura 1).

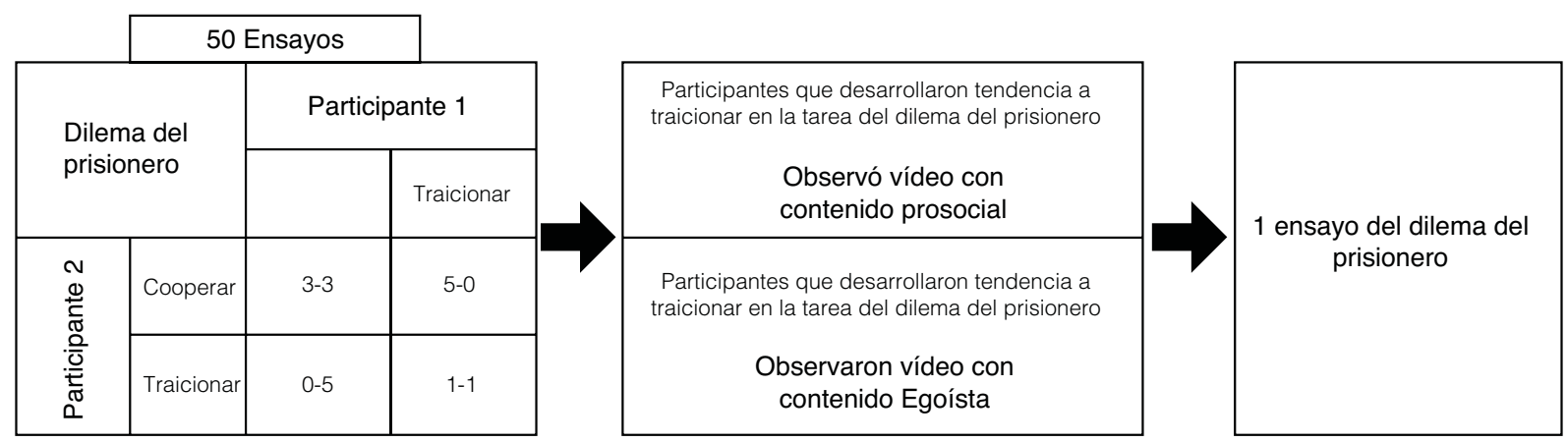

Figura 1 | Esquema de orden del instrumento

Durante la primera fase del experimento, los participantes fueron expuestos a 50 ensayos del dilema del prisionero. Un grupo de participantes fue expuesto a un confederado que cooperó en 45 de los 50 ensayos (compañero cooperativo). El otro grupo de participantes 
fue expuesto a un confederado que cooperó solo en 5 de los 50 ensayos (compañero egoísta). Esta primera parte toma alrededor de 20-30 minutos en desarrollarse.

Lo anterior buscaba favorecer una tendencia de comportamiento en los participantes. Aquellos participantes que cooperaron 25 ensayos o menos (sujetos que para efectos del estudio se entendieron como participantes "egoístas") fueron expuestos a un video en habla hispana en el cual se observaban unos niños compartiendo alimentos entre sí (video que muestra comportamiento "prosocial"). Los participantes que cooperaron 26 ensayos o más (sujetos que se entendieron como participantes "prosociales") fueron expuestos a un video en habla inglesa con subtítulos al español del tráiler de la película "El lobo del Wall Street" (video que muestra personas comportándose de forma "egoísta"). Independientemente del video observado, la duración de la exposición del vídeo fue de 2 minutos.

Finalmente, luego de la presentación del video, los participantes tuvieron un único ensayo del dilema del prisionero, con el propósito de observar el cambio o no de la conducta en función del video. Dicho ensayo es igual a los presentados en la primera fase.

\section{Procedimiento}

La aplicación del software se realizó durante una sesión de una hora, en un salón con más de 20 computadoras, a los cuales cada participante fue asignado aleatoriamente. En cada computador el software ya se encontraba abierto para iniciar la actividad. Un miembro del equipo investigador dio la bienvenida a los participantes y les pidió que leyeran y si estaban de acuerdo firmaran el consentimiento informado. Luego, el investigador indicó cómo dar inicio al software y les pidió leer las instrucciones, las cuales se explicaron nuevamente por el investigador y si estas estaban claras se les daba la orden de iniciar. Al finalizar, se agradeció su participación y se les dio a conocer el objetivo de la investigación. La presente investigación se rigió por la ley 1090 que regula el ejercicio profesional del psicólogo; así mismo, se tuvo en cuenta la resolución 008430 que permitió clasificar la investigación con riesgo mínimo.

\section{Análisis de Datos}

Inicialmente, se calcularon los estadísticos descriptivos de las distintas variables incluidas en el estudio. Seguido a esto, con el objetivo de establecer el efecto de reciprocidad y debido a que la distribución de los datos no cumplió con el supuesto de normalidad, se $\in \| 52$ 
utilizó la prueba de U de Mann Whitney. Debido a que las variables eran categóricas se realizó el estadístico chi-cuadrado, con el fin de identificar si luego de la presentación del video los participantes cambiaron su tendencia de comportamiento.

\section{Resultados}

La prueba de normalidad Kolmogorov-Smirnov indica que no se cumple con el supuesto de normalidad necesario para realizar pruebas paramétricas. (Estadístico $=.237 ; \mathrm{gl}=69 ; \mathrm{p}>.05$ ). Con el fin de determinar si se presenta el efecto de reciprocidad a partir del comportamiento del confederado, se utilizó la prueba no paramétrica U de Mann-Whitney, la cual indicó que existen diferencias significativas en la media del porcentaje total de cooperaciones dependiendo del confederado al que estuvo expuesto el participante $(U=271.5 ; \mathrm{P} \leq .001)$, estableciendo que sí existe un efecto estadísticamente significativo de reciprocidad a partir del comportamiento del confederado.

Este efecto de reciprocidad se confirma al observar la figura 2. Se evidencia un promedio mayor de cooperaciones para el grupo expuesto a un confederado prosocial (MD = 23.76; SD $=14.26)$, frente a un confederado egoísta (MD = 12.17; SD =9.47).

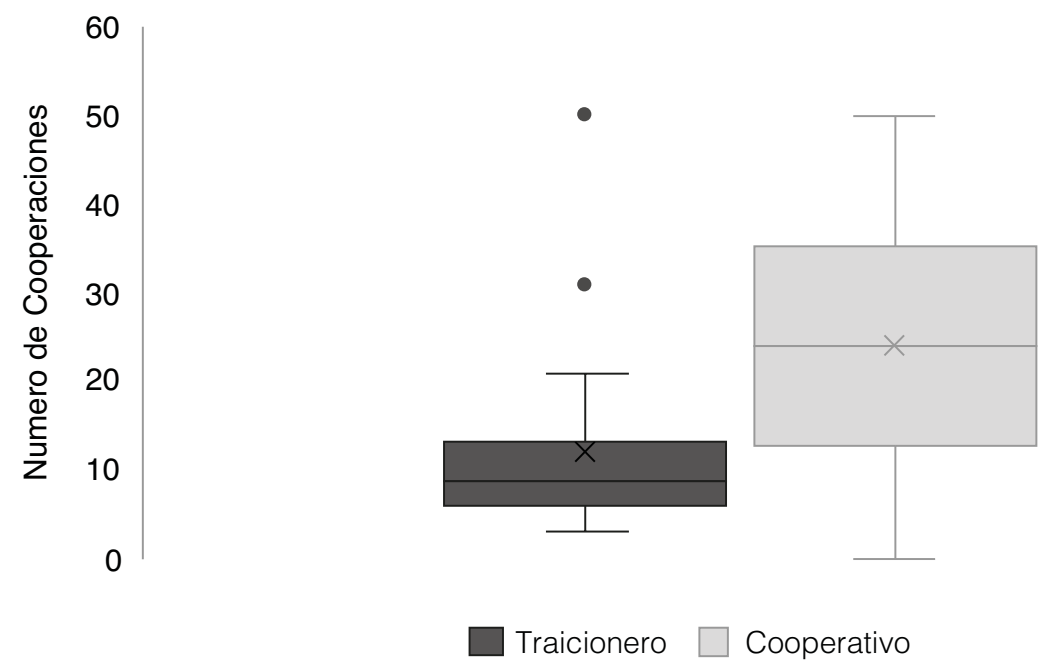

Figura 2. Promedio de cooperaciones según el confederado al que se estuvo expuesto. 
Para evaluar la relación de los videos con contenidos prosociales y egoístas, sobre el cambio o no de una tendencia previamente desarrollada, se realizó una prueba chi-cuadrado (Ver Tabla 1).

Tabla 1 | Tablas cruzadas entre la tendencia desarrollada y el último ensayo

\begin{tabular}{cccccc}
\hline & & \multicolumn{3}{c}{ Oneshot } & \\
\cline { 3 - 4 } & & & Traicionar & Cooperar & Total \\
\hline \multirow{2}{*}{ Tendencia } & Traicionar & Recuento & 18 & 32 & 50 \\
\hline \multirow{2}{*}{ Total } & Cooperar & Recuento & 5 & 14 & 19 \\
\hline & & Recuento & 23 & 46 & 69 \\
\hline
\end{tabular}

Los resultados indican que no existe una asociación significativa entre la presentación del vídeo y un cambio en la tendencia previamente desarrollada (; P > .05). Cabe mencionar que el $64 \%$ de las personas que tendieron a traicionar al ser expuestas a un video con contenido prosocial cambiaron su comportamiento después de la presentación de dicho video, optando por cooperar. En contraste, solo el $26 \%$ de las personas que tendieron a cooperar, traicionaron luego de observar un video con contenido egoísta.

\section{Discusión}

El primer objetivo de la investigación fue identificar el efecto de reciprocidad a partir del comportamiento egoísta/prosocial de un confederado sobre el comportamiento individual en ensayos reiterados del dilema del prisionero. Ribes-Iñesta (2008) sostiene que en situaciones en las que existen contingencias compartidas con otros individuos, el comportamiento propio tiende a la reciprocidad. Diferentes autores (Kerr, et al., 2009) sostienen que el comportamiento individual depende considerablemente del comportamiento de los demás y que ante tareas 
que impliquen la ganancia o la pérdida de un beneficio en común, el individuo tiende a comportarse de forma recíproca. En el presente estudio se observó que los participantes tendieron a cooperar cuando los otros participantes se comportaron de forma prosocial, y tendieron a actuar de forma egoísta al ser expuestos a compañeros con tendencia egoísta. Los resultados obtenidos soportan lo encontrado por los autores mencionados anteriormente.

El segundo objetivo del estudio fue identificar el efecto de la presentación de un video con contenido prosocial sobre el cambio o no de una tendencia a comportarse de forma egoísta. La revisión empírica relacionada con el comportamiento prosocial y videos con contenido similar evidencian un efecto significativo de vídeos con contenido prosocial sobre el comportamiento prosocial (Prot, et al., 2013) incluso, encontrándose en estudios longitudinales relaciones positivas y significativas a largo plazo entre dichas variables (PadillaWalker, Coyne, Collier \& Nielson, 2015). Los resultados del presente estudio indican que el $64 \%$ de los participantes que habían desarrollado una tendencia a traicionar en el dilema del prisionero, cooperaron después de ser expuestos a un video con contenido prosocial. Sin embargo, este resultado carece de significancia estadística, por lo tanto, se rechaza la segunda hipótesis de la investigación. El hecho de que este resultado haya sido contrario a lo reportado en estudios previos sobre comportamiento prosocial puede deberse a que en el contexto del experimento los participantes fueron expuestos a un único video con contenido prosocial.

En relación con el tercer y último objetivo, el cual corresponde a identificar el efecto de la presentación de un video con contenido egoísta sobre el cambio o no de una tendencia a comportarse de forma prosocial, no se ha evidenciado estudios en torno al efecto de videos con contenido egoísta. La evidencia empírica gira en torno a la presentación de videos con contenido violento, sin embargo, siendo el egoísmo una conducta donde el bienestar del otro se ve afectado, es posible considerar este tipo de conductas como violentas y opuestas a la prosocialidad (Tur \& Grande, 2009). Los resultados en torno a la presentación de videos violentos no han sido concluyentes, mientras que en algunos casos han demostrado tener un efecto significativo en el desarrollo de comportamiento agresivo, en otros casos no se ha evidenciado un efecto significativo de este tipo de videos (Cruz, 2014; Hoffman, 2014; Qian \& Da-Jun, 2014; Quiros \& Geovanna, 2016; Ramos, Ferguson \& Frailing, 2014). 
A partir de esta investigación se encontró que los videos con contenido egoísta no tienen un efecto significativo en una tendencia previamente desarrollada a cooperar, siendo que solo el $26 \%$ de las personas que tendieron a cooperar, traicionaron luego de observar un video con contenido egoísta. Los resultados obtenidos son similares a los hallazgos de Brummert \& Warburton (2011) los cuales buscaron comparar el efecto de la exposición a media auditiva y visual, de tipo violento, en el comportamiento de agresión, siendo que no se evidenciaron diferencias significativas entre los participantes que vieron videos musicales violentos y los que no. Así mismo, los resultados obtenidos por Ramos, Ferguson \& Frailing (2014) quienes buscaron establecer los efectos de videos con contenido violento en la conducta de cooperación por medio de una tarea del dilema del prisionero, evidencian la ausencia de efectos significativos de los videos violentos sobre la conducta cooperativa.

El hecho de que la mayoría de los participantes que tendieron a cooperar antes del video lo continuaron haciendo después del mismo; que la mayoría de los participantes que fueron egoístas cambiaron después del video y que la tendencia general posterior al video fue a cooperar, puede sugerir que ante tareas experimentales tipo dilemas sociales pueden favorecer interpretaciones por parte de los participantes hacia lo que es moralmente correcto en este tipo de situaciones (Cubitt, Drouvelis, Gächter \& Kabalin, 2011). Es posible que entender cómo el traicionar puede perjudicar al compañero pudiera derivar en disminuir el comportamiento de traicionar en la tarea. Para próximos estudios se recomienda indagar nociones morales de los participantes antes, durante y después de la exposición a dilemas morales.

Otro aspecto a resaltar, teniendo en cuenta que el entendimiento de la reciprocidad y el efecto de contenido audiovisual sobre el comportamiento tiende a ser estudiado individualmente, es que el presente estudio implementa tanto el dilema del prisionero, para identificar el efecto de reciprocidad, como los vídeos para identificar su efecto sobre una tendencia de comportamiento, en un solo experimento.

Es importante considerar ciertas limitaciones en el desarrollo de la presente investigación. En cuanto al tipo de videos utilizados, dado que no se hizo control de algunas variables tales como el idioma de los videos, el tipo de personajes involucrados (niños frente a adultos), entre otras, se sugiere homogeneizar los videos (Lienhart, Effelsberg \& Jain, 1998). Dada la dificultad expuesta respecto a la naturaleza de los vídeos y la relevancia social de 
promover comportamiento prosocial, otra recomendación estaría orientada al diseño metodológico, de modo que en vez de manipular dos variables independientes en dos grupos diferentes, se implemente únicamente una variable independiente y un grupo control; en ese sentido se podría desarrollar un estudio en el cual, luego de garantizar una tendencia a traicionar en el dilema del prisionero, se sugiere dividir al grupo que ha desarrollado dicha tendencia en dos, de modo que uno fuera un grupo experimental y otro un grupo control. Posterior a esto, exponer al grupo experimental a un vídeo que favorezca el comportamiento prosocial e indagar los efectos del video sobre el cambio o no de la tendencia a traicionar previamente desarrollada en la tarea.

\section{Referencias}

Attari, S. Z., Krantz, D. H., \& Weber, E. U. (2014). Reasons for cooperation and defection in real-world social dilemmas. Judgment and Decision Making, 9(4), 316-334. Recuperado de http://journal.sjdm. org/14/14517/jdm14517.pdf

Bandura, A., Ross, D., \& Ross, S. (1963). Imitation of film-mediated aggressive models. The Journal of Abnormal and Social Psychology, 66(1), 3-11. Doi:10.1037/h0048687

Baum, W. M., Paciotti, B., Richerson, P., Lubell, M. \& McElreath, R. (2012). Cooperation due to cultural norms, not individual reputation. Behavioural Processes, 91(1), 90-93. Doi:10.1016/j.beproc.2012.06.001

Brummert, H. I., \& Warburton, W. A. (2011). The effect of auditory versus visual violent media exposure on aggressive behavior: The role of song lyrics, video clips and musical tone. Journal of Experimental Social Psychology, 47(4), 794-799. Doi:10.1016/j.jesp.2011.02.006

Chen, X., Wu, H., Chen, H., Wang, L. \& Cen, G. (2001). Parenting Practices and Aggressive Behavior in Chinese Children. Parenting, Science and Practice, 1(3), 159-184. Doi: 10.1207/S15327922PAR0103_01

Corral, V. (2012). Sustentabilidad y Psicología positiva: Una visión optimista de las conductas proambientales y prosociales. México: Manual Moderno.

Cruz, C. (2014). There is broad consensus: Media researchers agree that violent media increase in children, and pediatricians and parents concur. Psychology of popular media culture. 4 (3). 200-214. Recuperado de: Doi:10.1037/ppm0000046 
Cubitt, R., Drouvelis, M., Gächter, S., \& Kabalin, R. (2011). Moral judgments in social dilemmas: How bad is free riding? Journal of Public Economics, 95(3-4), 253-264. Doi:10.1016/j.jpubeco.2010.10.011

Devilly, G. J., Brown, K., Pickert, I., \& O’Donohue, R. (2015). An Evolutionary Perspective on Cooperative Behavior in Gamers. Psychology of Popular Media Culture. Doi:10.1037/ppm0000097

Hoffman, A. (2014). Violent media games and aggression- Is it really time for a mea culpa? American psychologist. 69 (3), 305-314, Doi: 10.1037/a0035289

Hogan, M. (2012). Prosocial effects of media. Pediatric Clinics of North America, 59, 635-645. Doi: 10.1016/j. pcl.2012.03.020

Hoyos, L. (2013). Cooperación, solidaridad y egoísmo racional. Acerca de la relación entre moralidad y racionalidad. Revista de Estudios Sociales, (46), 24-30. Doi:10.7440/res46.2013.03

Kanazawa, S., \& Fontaine, L. (2013). Intelligent People Defect More in a One-Shot Prisoner's Dilemma Game. Journal of Neuroscience, Psychology, and Economics, 6(3), 201-213. Doi: 10.1037/npe0000010

Kerr, N., Rumble, A., Park, E., Ouwerkerk, J., Parks, C., Gallucci, M. \& Van Langue, P. (2009). How many bad apples does it take to spoil the whole barrel? Social exclusion and toleration for bad apples. Journal of Experimental Social Psychology, 45(4), 603 -613. Doi: 10.1016/j.jesp.2009.02.017

Lienhart, R., Effelsberg, W. \& Jain, R. (1998). VisualGREP: A systematic method to compare and retrieve video sequences. Storage and Retrieval for Image and Video Databases VI. 3312. Recuperado de: http:// citeseerx.ist.psu.edu/viewdoc/download?doi=10.1.1.23.6940\&rep=rep1\&type=pd

O'brien, D. (2012). Managing the urban commons. Human Nature: An Interdisciplinary Biosocial Perspective, 23(4), 467-489. Doi: 10.1007/s12110-012-9156-6

Padilla-Walker, L., Coyne, S., Collier, K., \& Nielson, M. (2015). Longitudinal Relations Between Prosocial Television Content and Adolescents' Prosocial and Aggressive Behavior: The Mediating Role of Empathic Concern and Self-Regulation. Developmental Psychology, 51(9), 1317-1328. Doi: 10.1037/a0039488

Parra-García, E., Nieto, J., \& Sánchez-Carrasco, L. (2014). Elección en palomas: Un análisis a través del dilema del prisionero. acta comportamentalia, 22(3), 247-257. Recuperado de http://pepsic.bvsalud.org/pdf/actac/v22n3/a01.pdf

E 58 
Prot, S., Gentile, D.A., Anderson, C.A., Suzuki, K., Swing, E., Ming Lim, K., Horiuchi, Y., Jelic, M., Krahé, B., Liuqing, W., Liau, A.K., Khoo, A., Petrescu, P.D., Sakamoto, A., Tajima, S., Andreea Toma, R., Warburton, W., Zhang, X. \& Pan Lam, B.C. (2013). Long-Term Relations Among Prosocial-Media Use, Empathy, and Prosocial Behavior. Psychological Science, 25(2), 358-367. Doi: 10.1177/0956797613503854

Qian, Z. \& Da-Jun, Z. (2014). The effects of viewing violent movie via computer on aggressiveness among college students. Computers in Human Behavior, 35, 320-325. Doi:10.1016/j.chb.2014.03.008

Quiros, E. \& Geovanna, J. (2016). Influencia de los programas televisivos violentos en el comportamiento agresivo de los niños de 7 a 8 años de edad en la escuela Assad Bucaram de la ciudad de Guayaquil, periodo lectivo 2016-2017. Recuperado de: http://repositorio.ulvr.edu.ec/handle/44000/439/browse?type=title

Ramos, R.A., Ferguson, C.J. \& Frailing, K. (2014). Violent Entertainment and Cooperative Behavior: Examining Media Violence Effects on Cooperation in a Primarily Hispanic Sample. Psychology of Popular Media Culture, 5(2). Doi:10.1037/ppm0000041

Redondo, J., Rueda, S., \& Amado, C. (2013). Conducta prosocial: una alternativa a las conductas agresivas. Investigium ire: Ciencias Sociales y Humanas, 4(1). Recuperado de: investigiumire.iucesmag.edu.co/ire/ index.php/ire/article/download/56/55

Ribes-Iñesta, E. (2010). Social Interactions. Conceptual Reflections and an experimental approach. En Schawrzer, R y French, P. (Eds). Personality, Human Development and Culture: International Perspectives on psychological Science. New York: International Union of Psychological Science.

Ribes-Iñesta, E., Rangel, N. \& Lopez-Valadéz, F. (2008). Análisis teórico de las dimensiones funcionales del comportamiento social. Revista Mexicana de Psicología, 25(1), 45-57. Recuperado de http://www.redalyc. org/articulo.oa?id=243016300003

Ribes-Iñesta, E., Rangel, N., Zaragoza, A., Magaña, C., Hernández, H., Ramírez, E., \& Valdez, U. (2006). Effects of differential and shared consequences on choice between individual and social contingencies. European Journal of Behavior Analysis, 7(1), 41-56. Recuperado de http://www.academia.edu/11677889/ Effects_of_differential_and_shared_consequences_on_choice_between_individual_and_social_contingencies 
Silva, G. (2014). Castigos y recompensas como mecanismos evolutivos para superar los dilemas sociales. Recuperado de: http://www.bdigital.unal.edu.co/45330/1/79862410.2014.pdf

Thøgersen, J. (2008). Social norms and cooperation in real-life social dilemmas. Journal of Economic Psychology, 29(4), 458-472. Doi:10.1016/j.joep.2007.12.004

Tur, V. \& Grande, I. (2009). Violencia y prosocialidad en los contenidos televisivos infantiles visionados por menores en Alicante. Zer, 14(27), 33-59. Recuperado de https://rua.ua.es/dspace/bitstream/10045/14442/3/ zer27-7-tur.pdf

Van Miltenburg, N., Buskens, V., Barrera, D. \& Raub, W. (2014). Implementing punishment and reward in the public goods game: the effect of individual and collective decision rules. International Journal of the Common, 8(1), 47-78. Doi: 10.18352/ijc.426/ 